\title{
A new effective method to estimate the effect of laser shock peening
}

\author{
Xianqian Wu, Chenguang Huang*, Xi Wang, Hongwei Song \\ Key Laboratory for Hydrodynamics and Ocean Engineering, Institute of Mechanics, Chinese Academy of Sciences, Beijing 100190, People's Republic of China
}

\section{A R T I C L E I N F O}

\section{Article history:}

Received 5 June 2010

Received in revised form

10 November 2010

Accepted 13 November 2010

Available online 20 November 2010

\section{Keywords:}

Laser shock peening

Plastically affected depth

Residual stress

Coupling analysis

Dimensional analysis

\begin{abstract}
A B S T R A C T
The paper focuses on the influencing parameters to the plastically affected depth and maximum residual stress of the metallic target after laser shock peening. Firstly, by using a new coupling analysis method, the shock pressure characteristics including peak pressure and pressure duration are given. Secondly, based on the deduced pressure profile, dimensional analysis method is employed to find the controlling parameters, and the relationships of plastically affected depth, maximum residual stress versus peak pressure, pressure duration and laser spot size are given. Thirdly, a two dimension axisymmetric finite element model based on LS-DYNA package is built, and the dynamic responses of metallic target subject to laser shock processing are computed with different input parameters. The result shows that the plastically affected depth is proportional to pressure duration, whereas the maximum residual stress is independent with it; the plastically affected depth and the maximum residual stress are not affected by laser spot size within a certain range, whereas have approximate linear relationships with peak pressure after reaching to a certain level; maximum residual stress and plastically affected depth increase significantly for a thin target configuration in laser shock peening system.
\end{abstract}

(C) 2010 Elsevier Ltd. All rights reserved.

\section{Introduction}

Laser shock peening (LSP) is an advanced surface enhancement technique for metallic materials to improve fatigue, corrosion and wearing resistance [1,2]. In LSP process, high-amplitude shock waves are generated through rapid expansion of high-temperature plasma induced by the interaction between high power density laser pulse and the material surface. While the shock wave propagates into the material, plastic deformation occurs to a depth where the stress no longer exceeds the Hugoniot elastic limit of the material, which induces residual stresses throughout the affected depth [3-5]. The plastically affected depth $L_{\mathrm{p}}$ and maximum residual stress $\sigma_{\mathrm{m}}$ are two basic parameters to evaluate the effects of LSP process. As a practical technique, most of the research work in this field focused on experiments on various metallic alloys, while some comprehensive modeling capacities based on analytical models and dynamic finite element models have been established to simulate LSP in the last few decades [6-15]. The shock pressure profile, which affects $L_{\mathrm{p}}$ and $\sigma_{\mathrm{m}}$, is very important for the effects of LSP. Fabbro et al. [16] established a physical method to predict the peak pressure based on the energy conservation. Sollier et al. [17] introduced the mass conservation into Fabbro's model, in which some variables of plasma can be analyzed. Recently, Wu and Yung [18] proposed a self-

\footnotetext{
* Corresponding author. Tel.: +8610 82543839 .

E-mail address: huangcg@imech.ac.cn (C. Huang).
}

closed thermal model considering most of physical processes. The effects of material constitutive model and strain rate on the shock pressure profile are not considered in those researches though. Furthermore, as LSP is a complex process with multiple variables, such as the pressure pulse magnitude and its profile, spot size and shape, material parameters and so on, an effective method is needed to predict the effect of LSP. Hu et al. [19] studied the parameters effect on residual stress in LSP based on numerical simulations and orthogonal experiments. Gulshan [20] proposed an optimization method to deal with the mixed-variable problem like LSP.

The article is organized as follows. A new coupling analysis is firstly proposed to get the characteristics of shock pressure profile. Then, the influences of LSP parameters such as peak pressure, laser radius and laser duration on the plastically affected depth and maximum residual stress are systematically analyzed using the dimensional analysis and dynamic finite element method.

\section{Pressure analysis}

LSP can usually be decoupled into two sequential physical processes: (1) pressure is firstly generated by the interaction of laser and coating confined by overlay materials and (2) shock wave propagates in the target material and induces plastic deformation and residual stress. Obtaining the pressure profile is the first step in simulating LSP process. In the previous models [16-18], a linear relationship between the shock pressure and the particle velocity is assumed, i.e. $p=Z u$, where $Z=\rho c$ is equivalent impedance and $u$ is 
boundary particle velocity. The material related effects to the shock pressure profile are not considered in those models therefore the constant $Z$ is taken. For LSP, assuming uniaxial strain state [11], the stress wave velocity can be written as

$c=\sqrt{\frac{(1-\nu)}{\rho(1+\nu)(1-2 \nu)} \frac{\mathrm{d} \sigma}{\mathrm{d} \varepsilon}}$,

so the equivalent impedance can be derived as

$Z_{i}=\sqrt{\frac{\left(1-\nu_{i}\right) \rho_{i}}{\left(1+\nu_{i}\right)\left(1-2 \nu_{i}\right)} \frac{\mathrm{d} \sigma_{i}}{\mathrm{~d} \varepsilon_{i}}}$

where $\nu_{i}$ and $\rho_{i}, i=1,2$ are Poisson's ratio and density for target and transparent overlay respectively. This indicates that the equivalent impedance $Z$ is associated with material constitutive model and strain rate, and therefore stress state related. From this point of view, a new coupling analysis method of the pressure is proposed, in which the effect of material constitutive model and strain rate is considered by taking a nonlinear relationship between the particle velocity and the pressure. The particle velocity depends not only on the pressure value, but also the pressure change rate.

Considering a Gaussian shape laser pulse with irradiation time $20 \mathrm{~ns}$, the plasma is assumed to be generated instantaneously with the initial length $L_{0}$ and the pressure is uniform in the plasma. The plasma expands with length $L(t)$. The particle velocities at the target boundary and the transparent overlay boundary are $u_{1}$ and $u_{2}$ respectively, which depend on the pressure $p$ and the pressure change rate $\mathrm{d} p / \mathrm{d} t$. The solution time is set to $50 \mathrm{~ns}$, in which there are two stages namely laser irradiation stage during irradiation time and plasma's adiabatic cooling stage during the remaining solution time.

For $t \leq 20 \mathrm{~ns}$, energy conservation is employed at this stage.

Mass conservation equation

$\frac{\mathrm{d} L(t)}{\mathrm{d} t}=u_{1}+u_{2}$

Energy conservation equation

$I(t)=p(t) \frac{\mathrm{d} L(t)}{\mathrm{d} t}+\frac{\mathrm{d}}{\mathrm{d} t}\left[E_{\mathrm{i}}(t) L(t)\right]$

where $E_{\mathrm{i}}(t)$ is the internal energy of plasma per unit volume. The first item of Eq. (2) is the work of pressure, and the second is the increase of internal energy. Considering the thermal energy $E_{\mathrm{T}}(t)$ takes a constant fraction $\alpha$ of internal energy and the other fraction of the internal energy being used to generate plasma, pressure $p(t)$ is related to the internal energy $E_{\mathrm{i}}(t)$ by the relation

$p(t)=\frac{2}{3} E_{\mathrm{T}}(t)=\frac{2}{3} \alpha E_{\mathrm{i}}(t)$

Eq. (2) can be written as

$I(t)=p(t) \frac{\mathrm{d} L(t)}{\mathrm{d} t}+\frac{3}{2 \alpha} \cdot \frac{\mathrm{d}}{\mathrm{d} t}[p(t) L(t)]$

Boundary conditions

$u_{1}=f_{1}\left(p, \frac{\mathrm{d} p}{\mathrm{~d} t}\right), \quad u_{2}=f_{2}\left(p, \frac{\mathrm{d} p}{\mathrm{~d} t}\right)$

where $f_{1}$ and $f_{2}$ are the certain functions of particle velocity of the transparent overlay and target material with applied pressure and its change rate respectively.

For $20 \mathrm{~ns}<t<50 \mathrm{~ns}$, the laser beam is switched off, and the plasma is considered as adiabatic cooling. The mass conservation equation and the boundary conditions are same as Eqs. (1) and (5) in the first stage; and the adiabatic cooling equation of plasma is taken.

$p(t)=p(\tau)\left(\frac{L(\tau)}{L(t)}\right)^{\gamma}$

where $p(\tau)$ and $L(\tau)$ are the pressure and length of plasma at the end of the first stage.

The analysis couples the expansion of the plasma and the deformation of the target material and its thickness effect. The analysis procedure for two different stages of the plasma is shown in Fig. 1. After getting the relationship between the particle velocity and the pressure for the target and transparent overlay by using LS-DYNA and LS-propostd for every time step, pressure value can be obtained by finite differential equations (1), (4) and (5) or (1), (5) and (6) for different stages at the next time step.

The coupling pressure analysis method is validated with experimental results [6] as Fig. 2. It gives the relationship between peak laser power density and peak pressure under the irradiation of Gaussian shape laser with FWHM 25 ns. The experimental results are measured with VISAR technique; and the simulation results are calculated by using the present method in which $\alpha$ and $L_{0}$ take 0.2 and $10 \mu \mathrm{m}$ respectively. The analysis results have good agreement with the experimental results.

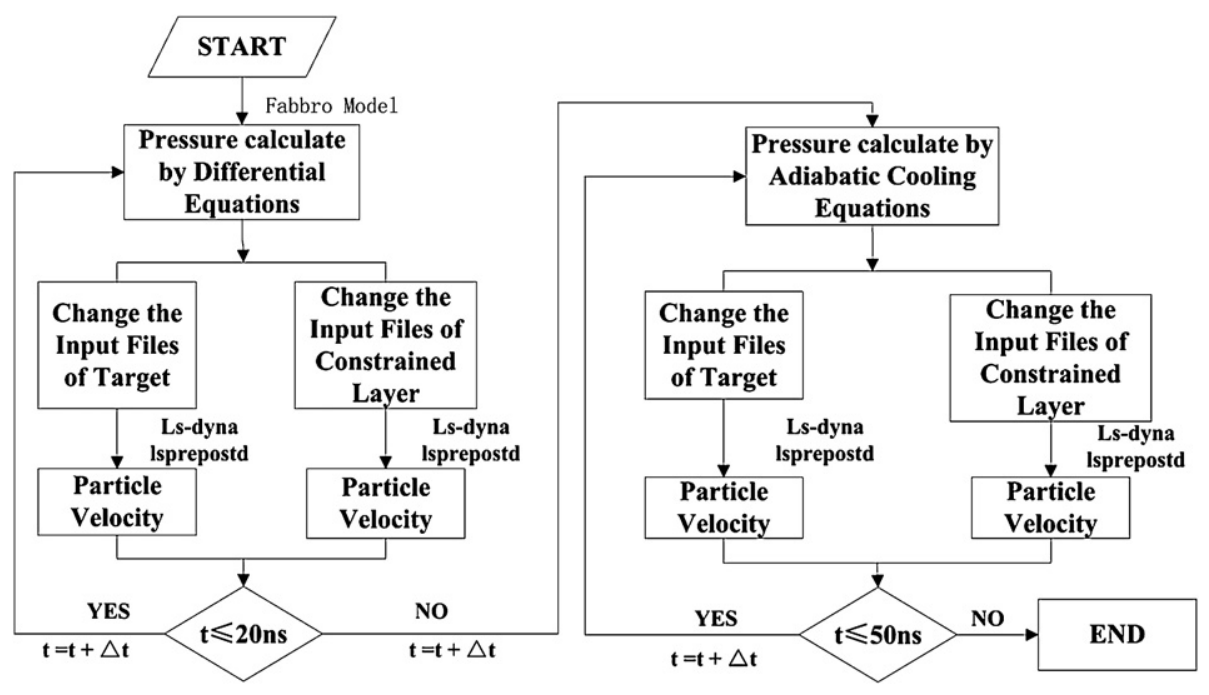

Fig. 1. Pressure analysis process with differential equations and LS-DYNA. 


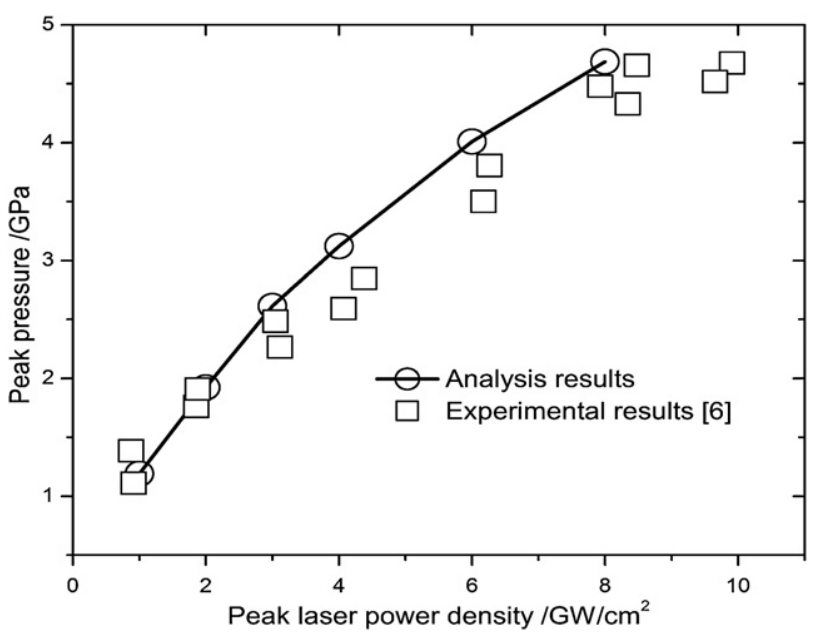

Fig. 2. The relationship between peak pressure and peak laser power density obtained from present analysis model and experiment respectively.

The calculated shock pressure profiles for different initial lengths and thermal internal energy ratios are shown in Fig. 3, where $L_{0}$ is initial length of plasma, and $\alpha$ is the thermal internal energy ratio. The results from Fabbro's pressure model are also included as a comparison. The peak pressure increases with decreasing the initial length and increasing the thermal internal energy ratio for both the present model and Fabbro's model. Also, the time to reach the peak pressure only depends on the initial length, and decreases with it for both models. This is probably because the analytical model neglects the formation process of the plasma. Because of the consideration of the interaction of elastic-plastic wave and nonconstant impedance, the peak pressure from the present analysis is larger than that of Fabbro's model. This difference decreases quickly while increasing the initial length from $1 \mu \mathrm{m}$ to $10 \mu \mathrm{m}$.

Compared with the previous models, it can be found that the model in the present research takes more factors into account. The expansion velocity of plasma is associated with not only its pressure state and material constitutive model of target and transparent overlay, but also the geometry model, such as the thickness of target and transparent overlay, to take the influence of free surface boundary into account. However, there are two variables $\alpha$ and $L_{0}$ in the present analysis model, which can be determined by considering the laser ablation and the evolution process of plasma.

\section{Dimensional analysis of LSP process}

The LSP process has many variables, such as the pressure pulse magnitude, spot size and shape, material parameters and so on. Dimensional analysis is a widely used method to solve this kind of problems with multiple variables. Through appropriate analysis of the relationship between dimensionless variables, the causality of physical phenomena can be highlighted [21].

According to the $\Pi$ theory of dimensional analysis [21], for a problem with $n$ independent variables $a_{1}, a_{2}, \ldots, a_{n}$, the dependent variable $a$ can be written as

$a=f\left(a_{1}, a_{2}, a_{3}, \ldots a_{k}, a_{k+1}, \ldots a_{n}\right)$

If there are $k$ independent dimensionless variables, $a_{1}, a_{2}, a_{3}, \ldots a_{k}$, then the following relations can be derived

$\frac{a}{\left(a_{1}^{m_{1}} a_{2}^{m_{2}} \ldots a_{k}^{m_{k}}\right)}=f\left(1,1, \ldots, 1, \frac{a_{k+1}}{\left(a_{1}^{p_{1}} a_{2}^{p_{2}} \ldots a_{k}^{p_{k}}\right)}, \frac{a_{k+2}}{\left(a_{1}^{q_{1}} a_{2}^{q_{2}} \ldots a_{k}^{q_{k}}\right)} \cdots \frac{a_{n}}{\left(a_{1}^{r_{1}} a_{2}^{r_{2}} \ldots a_{k}^{r_{k}}\right)}\right)$

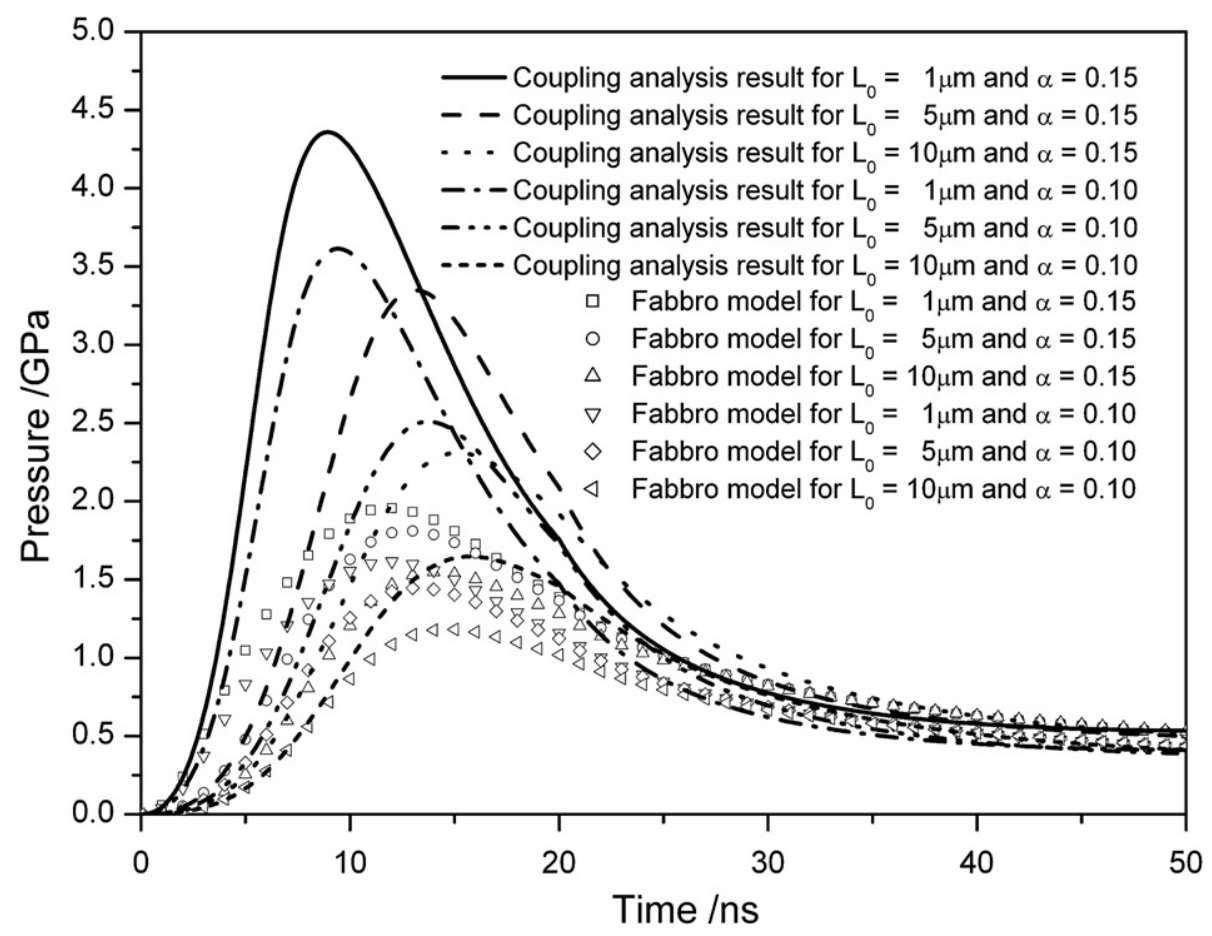

Fig. 3. Shock pressure profile with different initial plasma length $L_{0}$ and thermal internal energy ratio $\alpha$. 


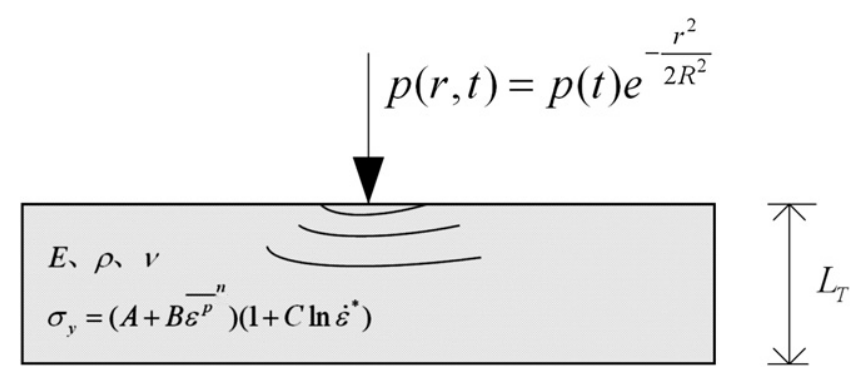

Fig. 4. The related parameters in dimensional analysis system.

It can be divided into two categories of variables in the LSP system (Fig. 4): pressure related parameters and material related parameters. The pressure related parameters are peak pressure $P$, pressure duration $\tau$, the radius of laser spot size $R$. For materials described with Johnson-Cook constitutive model [22], the effective stress can be written as

$\sigma_{\mathrm{y}}=\left(A+B \bar{\varepsilon}^{n}\right)\left(1+C \ln \dot{\varepsilon}^{*}\right)$

where $\overline{\varepsilon^{\mathrm{p}}}$ is equivalent plastic strain; $\dot{\varepsilon}^{*}=\overline{\varepsilon^{\mathrm{p}}} / \dot{\varepsilon}_{0}$ is normalized equivalent plastic strain rate and $\dot{\varepsilon}_{0}=1 \mathrm{~s}^{-1}, A, B, C$ and $m$ are material constants, and $n$ is the work hardening exponent.

The Material constitutive model related parameters are elastic modulus $E$, density $\rho$, Poisson's ratio $\nu$, constitutive parameters $A, B$, $n, C$ and equivalent plastic strain $\overline{\varepsilon p}$. The material geometry related parameter is the thickness of the target $L_{\mathrm{T}}$.

For a semi-infinite target, the thickness of the target $L_{T}$ is not taken into account in the analysis. The plastically affected depth $L_{\mathrm{p}}$ (the depth where radial compressive stress is zero) and maximum residual stress $\sigma_{\mathrm{m}}$ (maximum radial compressive stress) are functions of those parameters and can be written as

$L_{\mathrm{p}}=f\left(P, \tau, R, E, \rho, \nu, A, B, n, C, \overline{\varepsilon^{\mathrm{p}}}\right)$

$\sigma_{\mathrm{m}}=g\left(P, \tau, R, E, \rho, \nu, A, B, n, C, \overline{\varepsilon^{\mathrm{p}}}\right)$

If using Hugoniot elastic limit $\sigma_{\mathrm{H}}$ and the plastic modulus $E^{\prime}$ to describe the elastic and plastic dynamic behavior of the material, $\sigma_{\mathrm{H}}=(1-\nu) /(1-2 \nu) \sigma_{\mathrm{y}}, E^{\prime}=\mathrm{d} \sigma_{\mathrm{y}} / \mathrm{d} \overline{\varepsilon p}$, Eqs. (9) and (10) can be rewritten as

$L_{\mathrm{p}}=f\left(P, \tau, R, E, E^{\prime}, \rho, \sigma_{\mathrm{H}}\right)$

$\sigma_{\mathrm{m}}=g\left(P, \tau, R, E, E^{\prime}, \rho, \sigma_{\mathrm{H}}\right)$

With dimensionless analysis, there are four inherent dimensionless parameters $\sqrt{E^{\prime} / \rho} / \sqrt{E / \rho}, P / \sigma_{\mathrm{H}}, \sigma_{\mathrm{H}} / E$ and $(R / \sqrt{E / \rho}) \cdot(1 / \tau)$. Considering an unchanged material system, the material related dimensionless parameters $\sqrt{E^{\prime} / \rho} / \sqrt{E / \rho}$ and $\sigma_{\mathrm{H}} / E$ are excluded in the analysis. Consequently, three instinct relations can be obtained,

$$
\begin{aligned}
& L_{\mathrm{p}}=\sqrt{E / \rho} \cdot \tau \cdot f\left(\frac{P}{\sigma_{\mathrm{H}}}, \frac{R}{\sqrt{E / \rho}} \cdot \frac{1}{\tau}\right) \\
& L_{\mathrm{p}}=R \cdot f^{*}\left(\frac{P}{\sigma_{\mathrm{H}}}, \frac{R}{\sqrt{E / \rho}} \cdot \frac{1}{\tau}\right) \\
& \sigma_{\mathrm{m}}=P \cdot g\left(\frac{P}{\sigma_{\mathrm{H}}}, \frac{R}{\sqrt{E / \rho}} \cdot \frac{1}{\tau}\right)
\end{aligned}
$$

Eqs. (13) and (14) show the plastically affected depth $L_{\mathrm{p}}$ is proportional to pressure duration $\tau$ and laser spot radius $R$ when dimensionless parameters $P / \sigma_{\mathrm{H}}$ and $(R / \sqrt{E / \rho}) \cdot(1 / \tau)$ are constant; from Eq. (15), at the same condition, the maximum residual stress $\sigma$ is proportional to peak pressure $P$ and not affected by pressure duration $\tau$ and laser spot radius $R$.

\section{FEM simulations}

The three-dimensional simulation with LS-DYNA explicit and implicit method for single and multiple LSP has been proposed by $\mathrm{Hu}$ et al. [15]. With convergence mesh configuration, the simulation results are well correlated with the available experimental data. For a circular shaped laser spot, the 2D simulation is an efficient way because of less computational requirements [20]. A two-dimensional

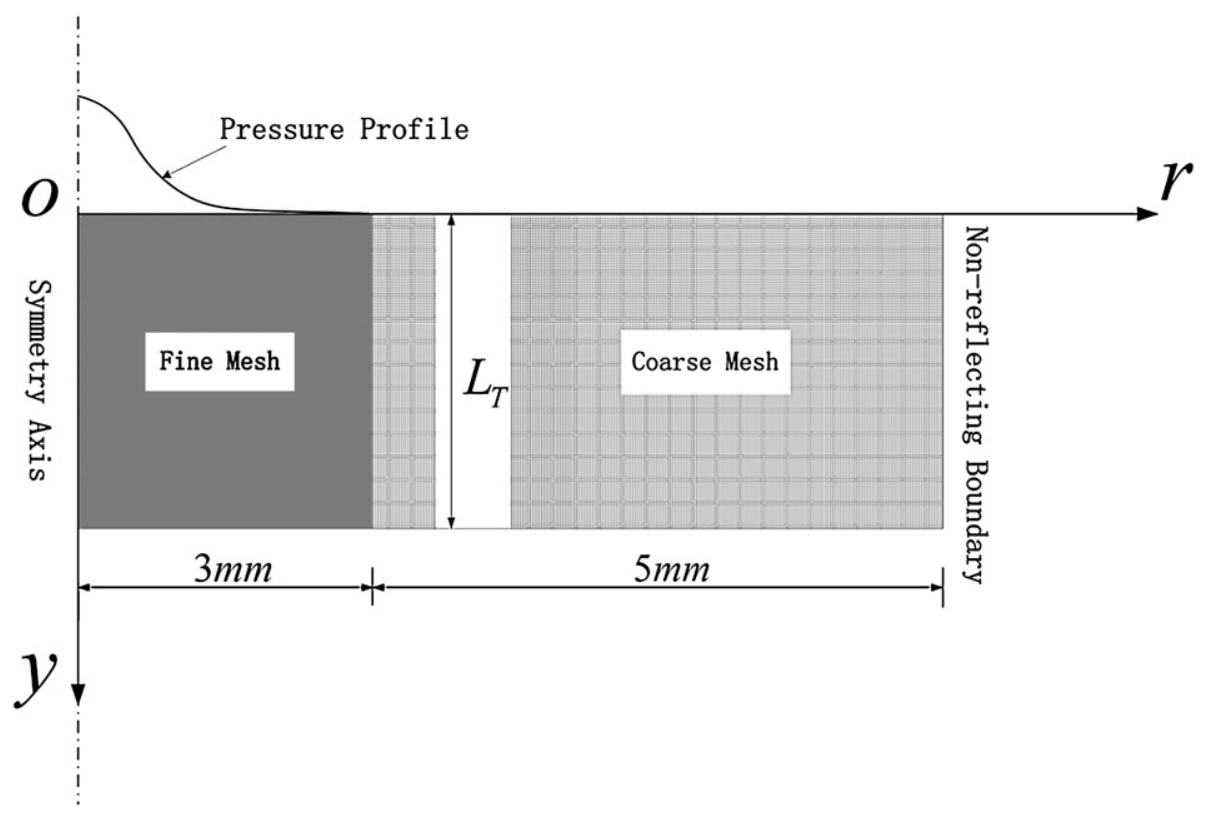

Fig. 5. The two-dimensional finite element simulation model. 


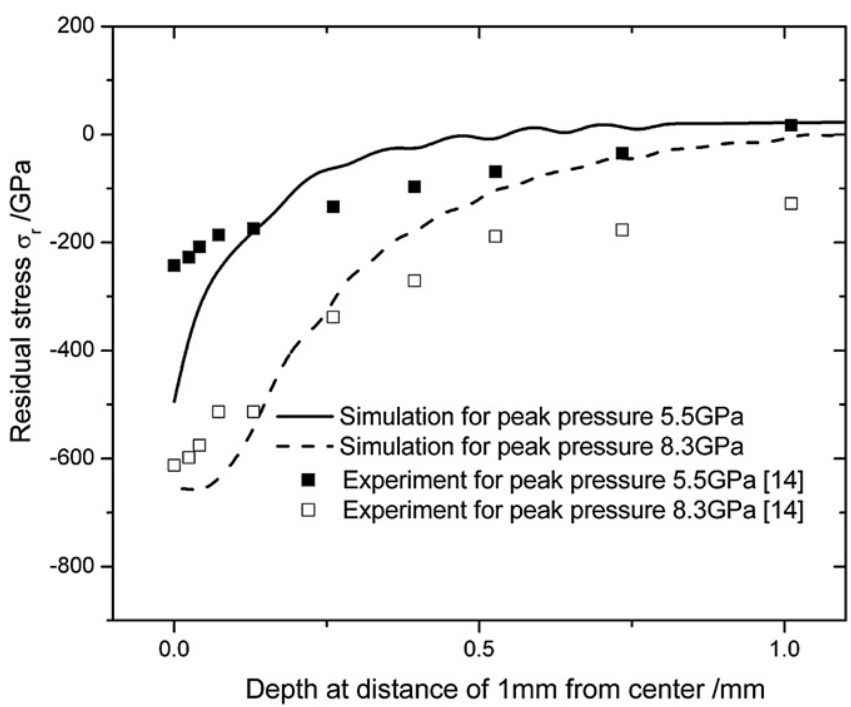

Fig. 6. Residual stress comparison between simulation and experiment data.

axisymmetric finite element model is developed using LS-DYNA package, and the dynamic responses of the metallic target without opaque overlay subject to LSP are simulated. The FE model represents a cylindrical target with a circular shaped laser spot and is depicted in Fig. 5. The model consists of a $3 \times \mathrm{L}_{\mathrm{T}} \mathrm{mm}^{2}$ fine meshed rectangular area with $120,0002 \mathrm{D}$ solid elements surrounded by $5 \times \mathrm{L}_{\mathrm{T}} \mathrm{mm}^{2}$ relative coarse meshed rectangular area with $120,0002 \mathrm{D}$ solid elements, where $L_{T}$ is the thickness of target. The Johnson-Cook constitutive model without considering the effect of temperature [20] is used.

Firstly, the simulation is conducted for Ti-6Al-4V alloy and compared with the experiment data as shown in Fig. 6. The simulation parameters used in our model and the experiment data are all from Amarchinta et al. [14]. The residual stress distributions for two different peak pressures were calculated. The simulation for peak pressure $8.3 \mathrm{GPa}$ matches with the experimentally determined stress profile especially at the region close to the surface, while the simulation for peak pressure 5.5 GPa shows much higher residual stress at the surface. Other than this, for the two peak pressures, the simulation is able to predict the trends for the entire depth of $1.0 \mathrm{~mm}$, indicating the consistency of the simulation model.

To validate the conclusions derived in Section 3, a parametric study was conducted using the model above. The distributed pressure, $p(r, t)=p(t) e^{-\frac{r^{2}}{2 R^{2}}}$, is applied on the upper boundary [23], where $R$ is the radius of laser spot, $p(t)$ is calculated from the coupling method in Section 2. The right boundary is non-reflected boundary condition to define unbounded domain. The computation time is set to $10,000 \mathrm{~ns}$ as suggested by Ding and Ye [24] to make sure the saturation of residual stress field. The 45 steel is chosen as the target material and the material properties used in the simulation are shown in Table 1.

Table 1

Basic material data for 45 steel [25].

\begin{tabular}{lc}
\hline Material properties, units & Value \\
\hline Young's modulus, $E[\mathrm{GPa}]$ & 206 \\
Poisson's ratio, $\nu$ & 0.3 \\
Density, $\rho\left[\mathrm{kg} / \mathrm{m}^{3}\right]$ & 7850 \\
Parameter $A[\mathrm{MPa}]$ & 507 \\
Parameter $B[\mathrm{MPa}]$ & 320 \\
Parameter $C[\mathrm{MPa}]$ & 0.064 \\
Parameter $n$ & 0.28 \\
\hline
\end{tabular}

Five groups of simulations are conducted and the parameters are shown as the following,

I. $P=4 \mathrm{GPa}$, and $(R / \sqrt{E / \rho}) \cdot(1 / \tau)$ is constant, $R=0.25 \mathrm{~mm}$, $\tau=10 \mathrm{~ns} ; \quad R=0.4 \mathrm{~mm}, \quad \tau=16 \mathrm{~ns} ; \quad R=0.5 \mathrm{~mm}, \quad \tau=20 \mathrm{~ns}$; $R=0.6 \mathrm{~mm}, \tau=24 \mathrm{~ns}$;

II. $P=4 \mathrm{GPa}, R=0.5 \mathrm{~mm}, \tau$ is $20 \mathrm{~ns}, 40 \mathrm{~ns}, 60 \mathrm{~ns}$ and $80 \mathrm{~ns}$ respectively;

III. $P=4 \mathrm{GPa}, \tau=20 \mathrm{~ns}, R$ is $0.1 \mathrm{~mm}, 0.25 \mathrm{~mm}, 0.4 \mathrm{~mm}, 0.5 \mathrm{~mm}$, $0.6 \mathrm{~mm}$ and $1 \mathrm{~mm}$ respectively;

IV. $\tau=20 \mathrm{~ns}, R=0.5 \mathrm{~mm}, P$ is $2.5 \mathrm{GPa}, 4 \mathrm{GPa}, 6 \mathrm{GPa}, 8 \mathrm{GPa}$ respectively;

V. $\tau=40 \mathrm{~ns}, R=0.5 \mathrm{~mm}, P=4 \mathrm{GPa}$, thickness $L_{\mathrm{T}}$ is $1 \mathrm{~mm}, 2 \mathrm{~mm}$, $3 \mathrm{~mm}, 4 \mathrm{~mm}, 5 \mathrm{~mm}, 6 \mathrm{~mm}$ respectively.

\section{Results and discussion}

Simulation results for case I are shown in Fig. 7. As shown in Fig. 7(a), the plastically affected depth $L_{\mathrm{p}}$ changes significantly with various $\tau$ and $R$ when the dimensionless parameter $(R / \sqrt{E / \rho}) \cdot(1 / \tau)$ keeps constant, that is consistent with Hu et al.'s result [19], whilst the maximum residual stress $\sigma_{\mathrm{m}}$ keeps at a similar level and changes slightly, which is consistent with Eq. (15). Fig. 7(b) shows
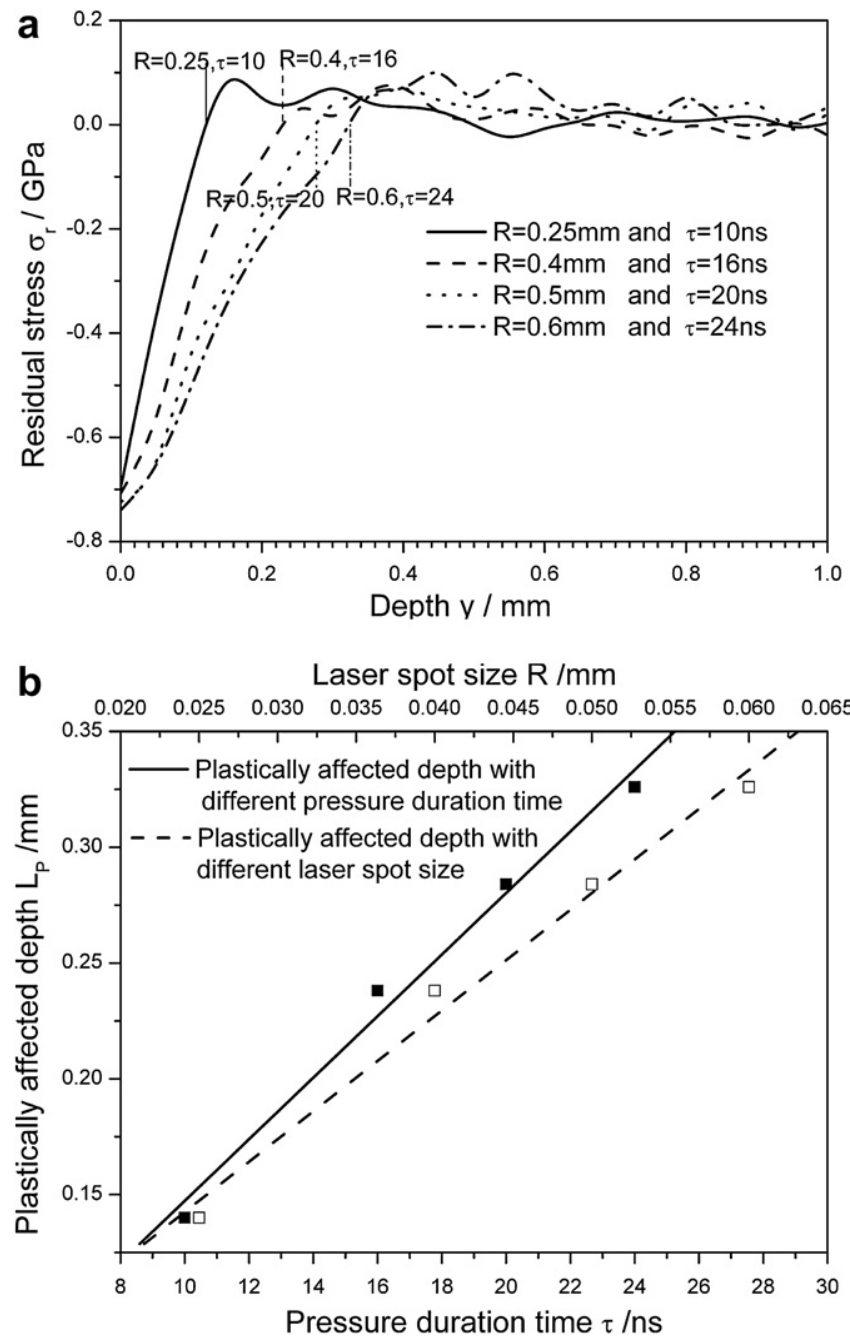

Fig. 7. Results of case I: (a) Distribution of $\sigma_{\mathrm{r}}$ in depth at the central location. (b) Relations between $L_{\mathrm{p}}$ and $\tau, R$. 
that $L_{\mathrm{p}}$ is linear with $\tau$ and $R$, that is in a good agreement with Eqs. (13) and (14). Because $R$ and $\tau$ change proportionally, the dependence of $L_{\mathrm{p}}$ on $\tau$ or $R$ cannot be determined uniquely just from this case and will be discussed in the next case.

Fig. 8 shows the simulation results for case II. From Fig. 8(a), $L_{\mathrm{p}}$ clearly increases for different pressure durations, but $\sigma_{\mathrm{m}}$ is almost unchanged. While $\tau$ varies from $20 \mathrm{~ns}$ to $80 \mathrm{~ns}, L_{\mathrm{p}}$ changes from $0.278 \mathrm{~mm}$ to $0.766 \mathrm{~mm}$, and $\sigma_{\mathrm{m}}$ keeps at about $720 \mathrm{MPa}$. From Fig. 8 (b), it can be found that $L_{\mathrm{p}}$ is proportional to pressure duration, and $\sigma_{\mathrm{m}}$ is almost constant with it. As $L_{\mathrm{p}}$ is linear with $\tau$ and $R$ that is derived at case I, and $L_{\mathrm{p}}$ is proportional to $\tau$ with different dimensionless parameter $(R / \sqrt{E / \rho}) \cdot(1 / \tau)$, while the material system unchanged, more simple relations can be derived as follows,

$L_{\mathrm{P}}=\sqrt{E / \rho} \cdot \tau \cdot f\left(\frac{\sigma_{\mathrm{H}}}{P}, \frac{R}{\sqrt{E / \rho}} \cdot \frac{1}{\tau}\right)=\sqrt{E / \rho} \cdot \tau \cdot f\left(\frac{\sigma_{\mathrm{H}}}{P}\right)$

$\sigma_{\mathrm{m}}=P \cdot g\left(\frac{P}{\sigma_{\mathrm{H}}}, \frac{R}{\sqrt{E / \rho}} \cdot \frac{1}{\tau}\right)=P \cdot g\left(\frac{P}{\sigma_{\mathrm{H}}}\right)$

Eq. (16) shows the plastically affected depth has linear relationship with pressure duration, while not affected by the laser
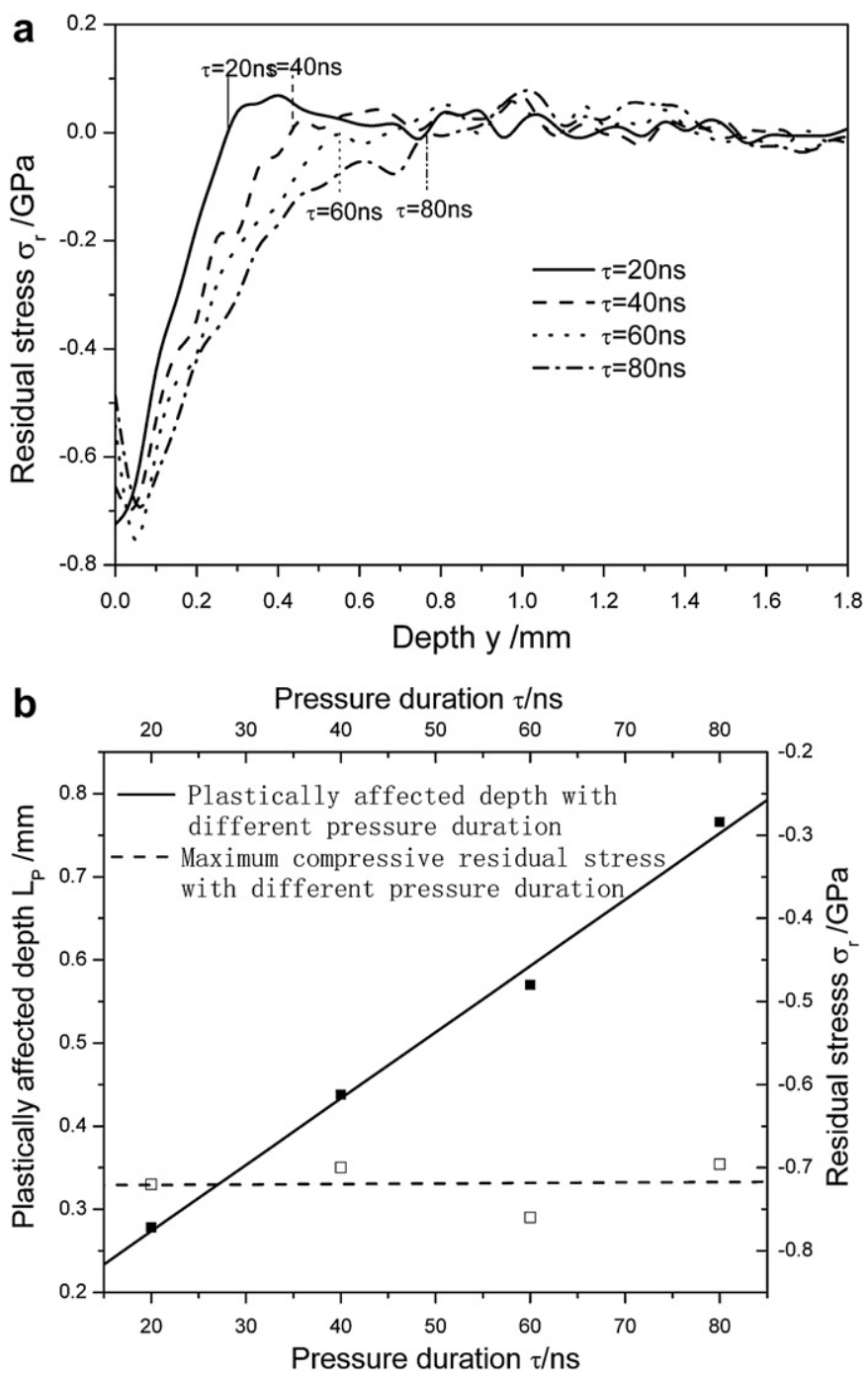

Fig. 8. Results of case II: (a) Distribution of $\sigma_{\mathrm{r}}$ in depth at the central location (b) Relations between $\sigma_{\mathrm{m}}, L_{\mathrm{p}}$ and $\tau$. spot size. Eq. (17) shows maximum residual stress is only affected by the peak pressure. For EPP materials, Ballard et al. [11,12] proposed an analytical solution of plastically affected depth, $L_{\mathrm{P}}=\left(C_{\mathrm{el}} C_{\mathrm{pl}} \tau /\left(C_{\mathrm{el}}-C_{\mathrm{pl}}\right)\right)\left(\left(P-\sigma_{\mathrm{H}}\right) / 2 \sigma_{\mathrm{H}}\right)$, where $C_{\mathrm{el}}$ and $C_{\mathrm{pl}}$ are elastic and plastic wave velocity. So the plastically affected depth is linear with pressure duration. Eq. (16) indicates that there is a similar conclusion for strain hardening and strain rate affected materials.

Fig. 9 shows the simulation results for case III. From Fig. 9(a), it shows that when $R$ exceeds $0.25 \mathrm{~mm}$, residual stress distributions in depth are almost identical, and when $R$ is smaller than $0.25 \mathrm{~mm}$, results change significantly. Fig. 9(b) shows that $L_{\mathrm{p}}$ slightly increases from $0.2 \mathrm{~mm}$ to $0.3 \mathrm{~mm}$ and $\sigma_{\mathrm{m}}$ slightly decreases from $700 \mathrm{MPa}$ to $750 \mathrm{MPa}$ when $R$ increases from $0.25 \mathrm{~mm}$ to $1 \mathrm{~mm}$. It is to say in that range of laser spot size, those results are consistent with Eqs. (16) and (17) which indicate that $L_{\mathrm{p}}$ and $\sigma_{\mathrm{m}}$ are not affected by $R$. But while $R$ is smaller than $0.25 \mathrm{~mm}, L_{\mathrm{p}}$ and $\sigma_{\mathrm{m}}$ decrease sharply. It is consistent with Peyre et al.'s result that the plastically affected depth could be strongly reduced with a small impact configuration [26]. This phenomenon can be ascribed to transverse unloading effect on the boundary of the laser spot when $R$ is not large enough compared with the thickness of material. The uniaxial strain assumption is not valid at such a condition that will be discussed in case $\mathrm{V}$.

Simulation results for case IV are shown in Fig. 10. Fig. 10(a) shows that $\sigma_{\mathrm{m}}$ and $L_{\mathrm{p}}$ are affected by the peak pressure $P$ largely, the
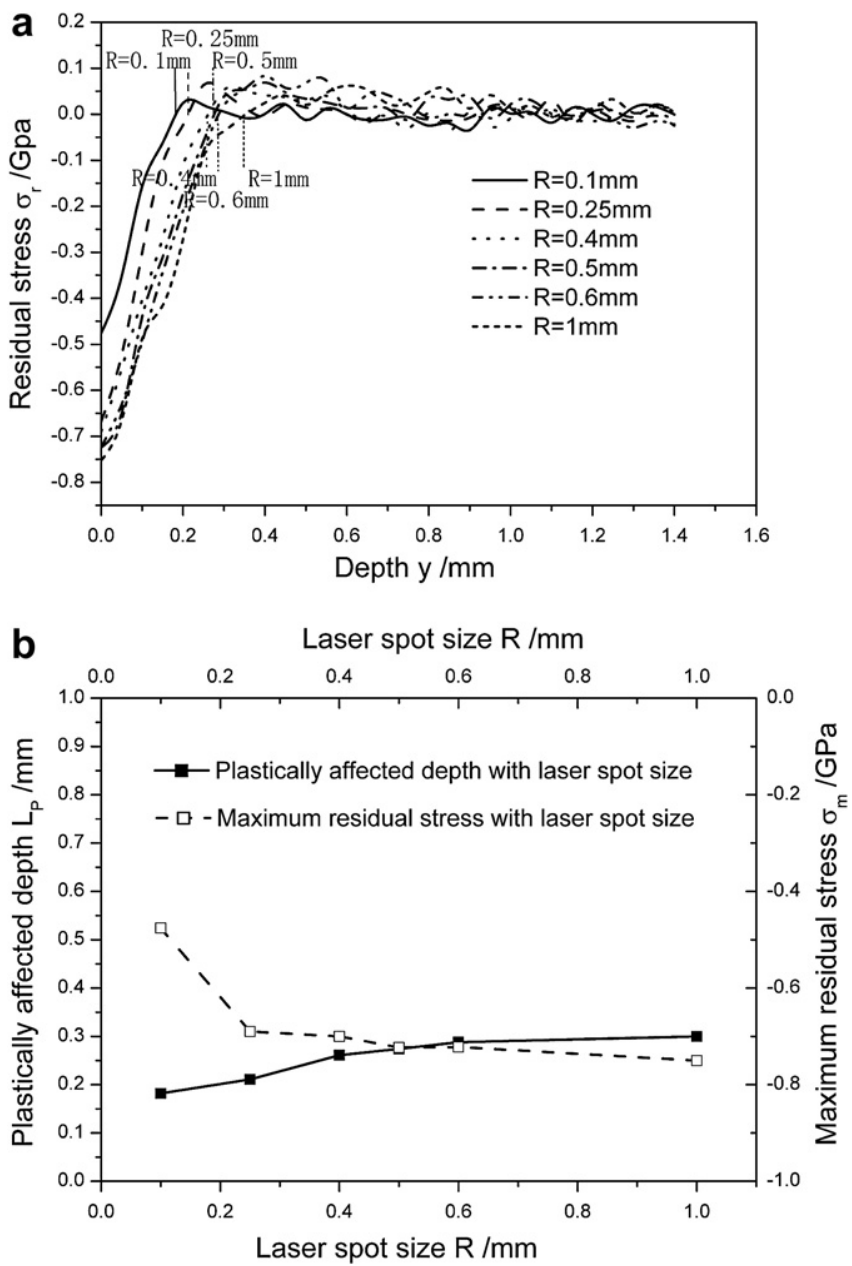

Fig. 9. Results of case III: (a) Distribution of $\sigma_{\mathrm{r}}$ in depth at the central location. (b) Relations between $L_{\mathrm{p}}, \sigma_{\mathrm{m}}$ and $R$. 

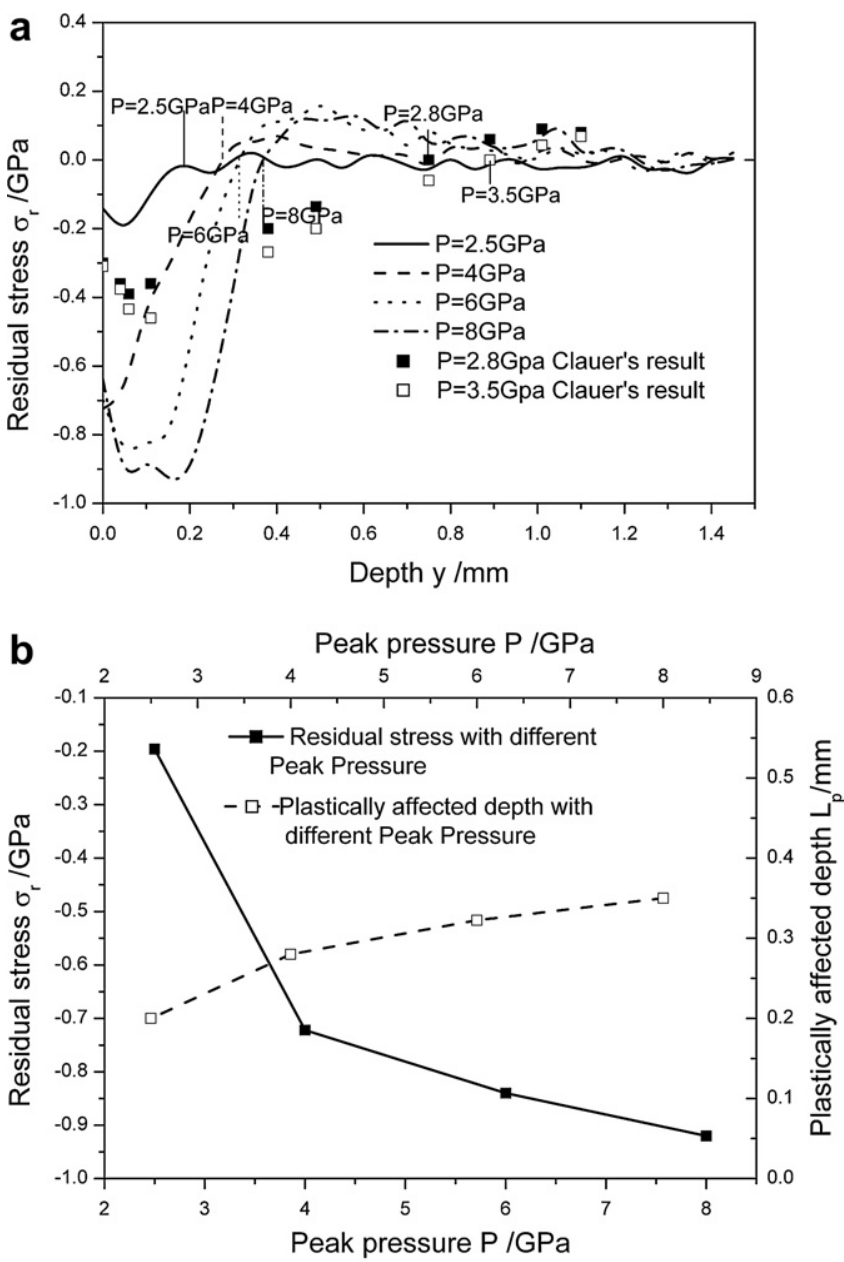

Fig. 10. Results of case IV: (a) Distribution of $\sigma_{\mathrm{r}}$ in depth at the central location. (b) Relations between $\sigma_{\mathrm{m}}, L_{\mathrm{p}}$ and $P$.

higher peak pressure, the larger $\sigma_{\mathrm{m}}$ and $L_{\mathrm{p}}$, which agrees with results of Clauer [8], Peyre et al. [26] and Hu et al. [19]. From Fig. 10(b), while the peak pressure exceeds $4 \mathrm{GPa}, \sigma_{\mathrm{m}}$ and $L_{\mathrm{p}}$ are almost linear with $P$. Maybe if peak pressure exceed $4 \mathrm{GPa}$ in this LSP system, the dimensionless parameter $P / \sigma_{\mathrm{H}}$ affected $\sigma_{\mathrm{m}}$ and $L_{\mathrm{p}}$ slightly, that is similar with Ballard et al.'s analytical solution [11,12].

The analysis above is based on a given pressure characteristics and the analyzed parameters are independent with each other. For the laser with identical energy and wavelength in the LSP system, different laser spot size and duration will lead to the change of the laser power density, which would change the shock pressure characteristics and consequently the effects of LSP. From Fabbro's equation, $P=A(\alpha /(2 \alpha+3))^{1 / 2} Z^{1 / 2} I^{1 / 2}$, where $A$ is absorption coefficient, $\alpha$ is the thermal internal energy ratio, $Z$ is equivalent impedance, and $I$ is laser powder density. For the same laser shock system, $I \propto J /\left(\tau R^{2}\right)$ and $p \propto I^{1 / 2}$, therefore $p \propto J^{1 / 2} /\left(\tau^{1 / 2} R\right)$. When the laser energy is constant, the shock pressure has inverse proportional relationship with $R$ and $\tau^{1 / 2}$. If the laser spot size decreases 50\%, the pressure will be doubled and twice of laser duration will lead to a decrease of $30 \%$ in the pressure. As shown in Eqs. (16) and (17), the change of laser spot and the pressure duration will affect the plastically affected depth and the maximum residual stress for a given laser energy system. Consistently, Fabbro et al. [27] and Peyre et al. [9] found the maximum surface residual stress could be achieved with a short laser pulse.

The influence of the target material thickness is shown in Fig. 11. It can be found that the residual stress distribution in depth is very similar with various material thicknesses from Fig. 11(a). And the
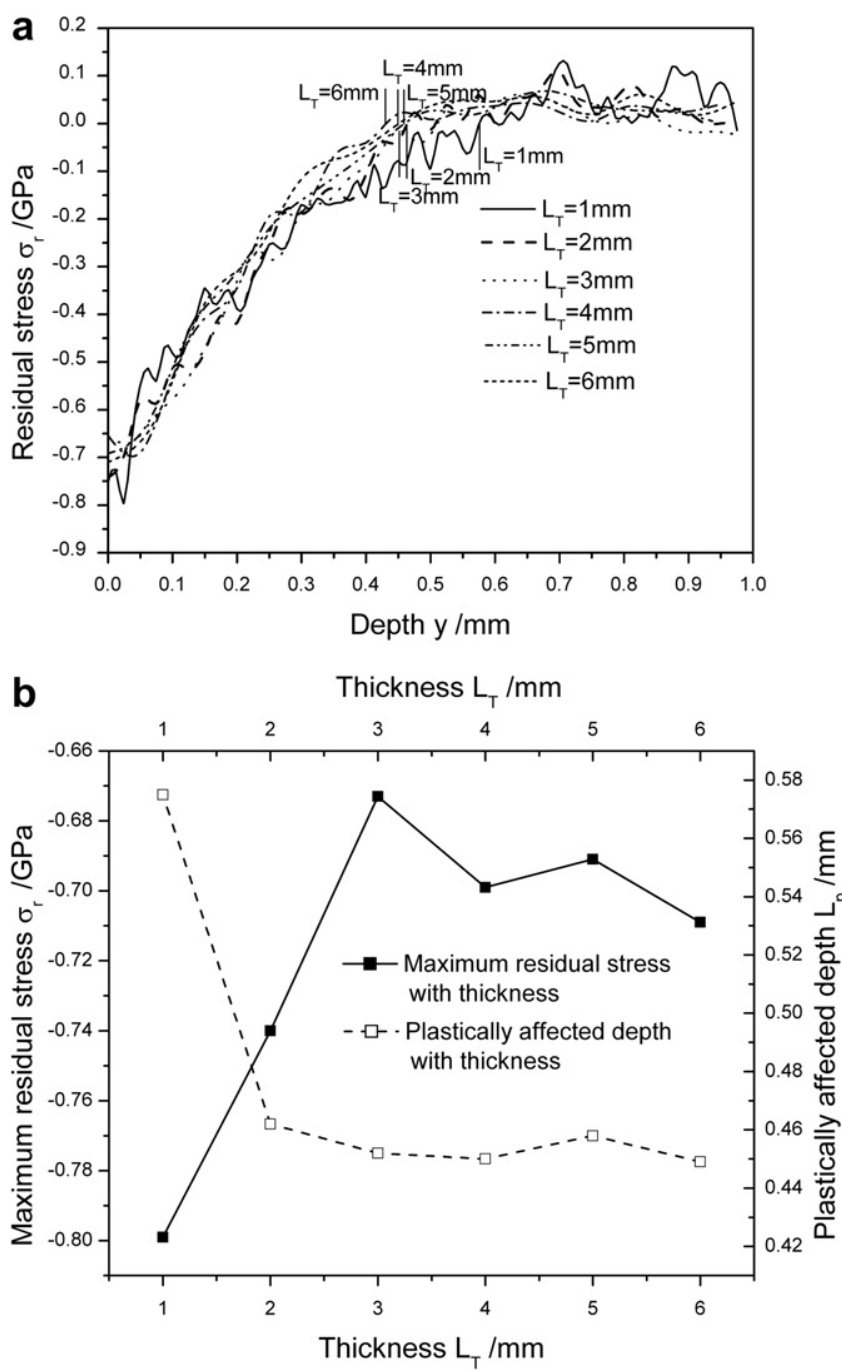

Fig. 11. Results of case V: (a) Distribution of $\sigma_{\mathrm{r}}$ in depth at the central location. (b) Relations between $\sigma_{\mathrm{m}}, L_{\mathrm{p}}$ and $L_{\mathrm{T}}$.

result with the smaller thickness flutters quickly that reveals that the complex interaction of stress waves in a thin material. Fig. 11(b) shows maximum residual stresses and plastically affected depths with different thicknesses. It shows obviously that the maximum residual stress and plastically affected depth change significantly when the thickness becomes smaller. The smaller the thickness is, the larger the maximum residual stress and plastically affected depth are. It also implies that a good shock effect can be achieved with a thin material configuration in LSP. But it may lead to unexpected deformation after the LSP process due to the relaxation of residual stress. The thin target must be constrained by some means.

\section{Conclusions}

In this paper, the dependence of the plastically affected depth and maximum residual stress on LSP process parameters are investigated by dimensional analysis and numerical simulations. The main conclusions are as follows:

1. An improved new coupling analysis method of shock pressure, which considering the nonlinear relationship between shock pressure and particle velocity and the effect of target thickness, is proposed. More experiments will be carried out to validate this method in the future. 
2. The dependent dimensionless parameters of the plastically affected depth and maximum residual stress are derived using dimensional analysis, which provide a possible method of systematic investigation for LSP process.

3. The major parameters $P / \sigma_{\mathrm{H}}$ and $(R / \sqrt{E / \rho}) \cdot(1 / \tau)$ that control the LSP effects are found for a given pressure characteristics with FEM simulations. The results are in good agreement with dimensionless equations.

4. The influence of material thickness is investigated by FEM analysis, which shows that, a good shock effect can be achieved with a thin material configuration. But the target must be constrained to avoid the relaxation of residual stress.

\section{Acknowledgements}

The authors thank the National Natural Science Foundation of China $(10972228,11002150)$ and Basic Research Equipment Project of Chinese Academy of Sciences (YZ200930) for financial support.

\section{References}

[1] Peyer P, Fabbro R. Laser shock processing: a review of the physics and applications. Opt Quantum Electron 1995;27:1213-29.

[2] Berthe L, Fabbro R, Peyre P, Tollier L, Bartnicki E. Shock waves from a waterconfined laser-generated plasma. J Appl Phys 1997;82:2826-32.

[3] Fairand BP, Clauer AH. Laser generated stress waves: their characteristics and their effects to materials. AIP Conf Proc 1979;50:27-42.

[4] Charles SM, Wei T, Ye L, Clark Graham, Mai Yiu-Wing. Laser shock processing and its effects on microstructure and properties of metal alloys: a review. Int J Fatigue 2002;24:1021-36.

[5] Zhang YK, Lu JZ, Ren XD, Yao HB. Effect of laser shock processing on the mechanical properties and fatigue lives of the turbojet engine blades manufactured by LY2 aluminum alloy. Mater Des 2009;30(5):1697-703.

[6] Berthe L, Fabbro R, Peyre P, Tollier L, Bartnicki E. Shock waves from a waterconfined laser-generated plasma. J Appl Phys 1997;82:2826-32

[7] Lu JZ, Luo KY, Zhang YK, Cui CY, Sun GF, Zhou JZ, et al. Grain refinement of LY2 aluminum alloy induced by ultra-high plastic strain during multiple laser shock processing impacts. Acta Mater 2010;58(11):3984-94.

[8] Clauer AH. Laser shock peening for fatigue resistance: proceedings of surface performance of titanium. Met Soc AIME; 1996:217-30.
[9] Peyer P, Berthe L, Fabbro R, Bartnicki E. Experimental determination by PVDF and EMV techniques of shock amplitudes induced by $0.6-3$ ns laser pulses in a confined regime with water. J Appl Phys 2000;33:498-503.

[10] Peyer P, Berthe L, Scherpereel X, Fabbro R. Experimental study of laser-driven shock waves in stainless steels. Appl Phys 1998;84:5985-92.

[11] Ballard P. Residual stresses induced by rapid impact - applications of laser shocking. Dr. Thesis, Fr.; 1991.

[12] Ballard P, Fournier J, Fabbro R, Frelat J. Residual stresses induced by lasershocks. J Phys IV 1991;C3:487-94.

[13] Hu YX, Yao ZQ Jun Hu. An analytical model to predict residual stress field induced by laser shock peening. J Manuf Sci Eng 2009;131(3):031017-24.

[14] Amarchinta HK, Grandhi RV, Langer K, Stargel DS. Material model validation for laser shock peening process simulation. Model Simul Mater Sci Eng 2009;17:015010-25.

[15] Hu YX, Yao ZQ Hu J. 3-D FEM simulation of laser shock processing. Surf Coat Technol 2006;201(3):1426-35.

[16] Fabbro R, Fournier J, Ballard P, Devaux D, Virmont J. Physical study of laserproduced plasma in confined geometry. J Appl Phys 1990;68:775-84.

[17] Sollier A, Berthe L, Peyre P, Bartnicki E, Fabbro R. Laser-matter interaction in laser shock processing. Int Soc Opt Eng 2003;4831:463.

[18] Wu BX, Yung CS. A self-closed thermal model for laser shock peening under the water confinement regime configuration and comparisons to experiments. J Appl Phys 2005;97:1135-52.

[19] Hu YX, Yao ZQ Wang F, Hu J. Study on residual stress of laser shock processing based on numerical simulation and orthogonal experimental design. Surf Eng 2007;23(6):470-8

[20] Gulshan S. Effective simulation and optimization of a laser peening process. Dr. Thesis, Wright State University; 2009.

[21] Tan QM. Dimensional analysis. 1st ed. Beijing: Chin. Univ. Sci. Technol. Press; 2005, pp. $12-14$

[22] Johnson G, Cook W. A constitutive model and data for metals subjected to large strains, high strain rates and high temperatures. In: Proceedings of the 7th international symposium on ballistics, The Hague, Netherlands; 1983. p. 541-7.

[23] Zhang W, Yao YL, Noyan IC. Microscale laser shock peening of thin films, Part 1: Experiment, modeling and simulation. J Manuf Sci Eng 2004;126(1):10-7.

[24] Ding K, Ye L. Three-dimensional dynamic finite element analysis of multiple laser shock peening processes. Surf Eng 2003;19(5):351-8.

[25] Chen G, Chen ZF, Tao JL, Niu W, Zhang QP, Huang XC. Investigation and validation on plastic constitutive parameters of 45 steel. Explos Shock Waves 2005;25(5):451-6.

[26] Peyre P, Berthe L, Scherpereel X, Fabbro R. Laser-shock processing of aluminium-coated 55C1 steel in water-confinement regime, characterization and application to high-cycle fatigue behaviour. J Mater Sci 1998;33:1421-9.

[27] Fabbro R, Peyre P, Berthe L, Scherpereel X. Physics and applications of lasershock processing. J Laser Appl 1998;10:265-79. 